\title{
Céline, Malaparte. Malaparte, Céline: una poetica
} del disincanto

Céline, Malaparte. Malaparte, Céline : une poétique du désenchantement

Céline, Malaparte. Malaparte, Céline: A Disillusioned Prose

Lorella Libeccio

\section{(2) OpenEdition}

\section{Journals}

Edizione digitale

URL: http://journals.openedition.org/cei/3424

DOI: $10.4000 /$ cei.3424

ISSN: 2260-779X

\section{Editore}

UGA Éditions/Université Grenoble Alpes

Edizione cartacea

ISBN: 978-2-84310-370-4

ISSN: 1770-9571

\section{Notizia bibliografica digitale}

Lorella Libeccio, «Céline, Malaparte. Malaparte, Céline: una poetica del disincanto», Cahiers d'études

italiennes [Online], 24 | 2017, online dal 28 février 2017, consultato il 26 mars 2021. URL: http:// journals.openedition.org/cei/3424 ; DOI: https://doi.org/10.4000/cei.3424

Questo documento è stato generato automaticamente il 26 mars 2021.

(c) ELLUG 


\title{
Céline, Malaparte. Malaparte, Céline: una poetica del disincanto
}

\author{
Céline, Malaparte. Malaparte, Céline : une poétique du désenchantement
}

Céline, Malaparte. Malaparte, Céline: A Disillusioned Prose

\section{Lorella Libeccio}

La grande défaite, en tout, c'est d'oublier, et surtout ce qui vous a fait crever, et de crever sans comprendre jamais jusqu'à quel point les hommes sont vaches. Quand on sera au bord du trou faudra pas faire les malins nous autres, mais faudra pas oublier non plus, faudra raconter tout sans changer un mot, de ce qu'on a vu de plus vicieux chez les hommes et puis poser sa chique et puis descendre. Ça suffit comme boulot pour une vie toute entière.

Louis-Ferdinand CÉLINE, Voyage au bout de la nuit.

Un uomo è una cosa ancora più triste e orrenda di questo mucchio di carne sfatta. Un uomo è orgoglio, crudeltà, tradimento, viltà, violenza. La carne sfatta è tristezza, pudore, paura, rimorso, speranza. Un uomo, un uomo vivo, è poca cosa, in confronto di un mucchio di carne marcia. Curzio MALAPARTE, La pelle.

\section{Due scrittori controversi}

1 Come ho voluto evocare attraverso il chiasmo del titolo, in questo saggio vorrei proporre un incrocio ideale tra Louis-Ferdinand Céline e Curzio Malaparte attraverso lo studio di tematiche concettualmente parallele. Proprio come in un chiasmo, si cercherà 
di costruire un discorso equilibrato attraverso la disposizione incrociata di alcuni argomenti della loro opera. Questa analisi speculare parte da un concetto interpretativo personale: secondo la chiave di lettura qui proposta, infatti, tra i due autori c'è un'analogia di punto di vista che consiste nella critica della società borghese e del capitalismo e nella loro volontà di illustrare la degradazione e la decadenza del mondo occidentale. Tali aspetti sono dei fulcri del loro pensiero, come un fil rouge che dà alla loro opera una forte coerenza logica.

2 Il mio articolo è dunque una proposta, un invito alla lettura e alla ricerca di assonanze e analogie nell'opera di due scrittori tanto diversi quanto complessi. Louis-Ferdinand Céline e Curzio Malaparte sono stati, infatti, attenti critici della società, degli intrecci di potere e politica, analisti della loro contemporaneità attraverso il lavoro saggistico e le loro opere letterarie, eternamente messi sotto accusa per il loro pensiero controverso e abilmente critico, se non volutamente contraddittorio.

3 Non si vuole di certo forzare un legame tra uomini tanto distanti per esperienze letterarie, politiche e di vita ma la loro diversità tanto palese non rende impossibile un tentativo di comparazione.

4 Partendo dal presupposto della loro appartenenza a un'epoca storica comune, presupposto imprescindibile per una comparazione non arbitraria, e cioè quella delle guerre mondiali e dei regimi dittatoriali, si proverà a tracciare un discorso che lasci spazio alla contraddittorietà della loro esperienza e alla complessità del periodo storico in cui la loro opera si inserisce.

5 Non si ha dunque la pretesa di spiegare le loro analogie fornendo delle risposte assolute ma cercando piuttosto di costruire un discorso che apra a stimoli futuri o che semplicemente dia spunti di riflessione sul loro lavoro. La pretesa classificatoria, non lasciando spazio alle innumerevoli incongruenze che ogni scrittore porta in sé, rischia di impoverire l'analisi. In tale ottica la ricerca vuole invece essere una delle tante possibilità di lettura del loro percorso politico ed estetico. L'autonomia della loro opera non è messa in dubbio in questa sede, non si vogliono dare risposte risolutive ma lasciar spazio al loro pensiero che, nonostante l'evidente trasformismo che li rende sfuggenti e difficilmente classificabili, mantiene in realtà una forte coerenza ${ }^{1}$.

6 Céline e Malaparte furono scrittori emblematici per la singolarità delle loro esistenze e il carattere peculiare della loro letteratura che si situa al centro dei dibattiti socioideologici della loro epoca; scrivere è un modo per definirsi attraverso il rifiuto e la solitudine, è il desiderio di un io che rigetta la società e vuole esprimere la propria denuncia attraverso la letteratura. Gli autori riusciranno quindi con questa loro pretesa di verità, di critica continua espressa senza alcuna moderazione, a farsi criticare, censurare e addirittura esiliare.

7 Il pensiero di Malaparte e Céline si basa su un'osservazione critica e attenta della società in cui vivono, osservazione che i due scrittori riescono a rendere attraverso una scrittura molto elaborata e personale. La loro critica va alla storia scritta dai potenti, nel tentativo di mettere in scena la tragedia della povertà e della sottomissione ai poteri invisibili, lasciando spazio a chi nella storia non ha nessuna autorità, buttando giù le false verità e la facile retorica. I due autori, di cui non è immediato trovare la chiave di interpretazione, propongono quindi una riflessione sul rapporto tra gli uomini, sulla relazione tra l'individuo e il potere politico-economico, sempre mostrando una coscienza della lingua e del loro ruolo di scrittori che li rende 
estremamente interessanti nel contesto letterario del primo Novecento e che non ha smesso di affascinare i critici sino ai giorni nostri.

Céline e Malaparte, come molti romanzieri loro contemporanei, esprimono il rifiuto del mondo, coscienti dell'impossibilità di salvarsi dal disastro che la guerra ha lasciato: l'uomo ha mostrato la sua crudeltà, si è denudato ed ogni forma di coesione e di unione è crollata. In tale contesto disastrato i due letterati si mostrano solitari, individualisti nelle loro scelte, avversari di ogni chiesa e partito. La loro è anche scrittura di immersione nel marcio della società, tra gli sventurati, attraverso uno stile singolare ed un lessico aspro e dirompente. C'è chi li amò, chi li criticò duramente, chi fu diviso tra l'ammirazione e la non condivisione delle loro scelte.

La loro disillusione si traduce inoltre in un certo odio per la felicità gratuita, per il successo esibito: «j'avais tout envie de vomir sur la vulgarité de son succès, de son orgueil $»^{2}$, in un rigetto della volgarità insita nella facile fortuna:

Non perché mi piaccia assistere allo spettacolo della miseria altrui, e dell'umiliazione, ma perché l'uomo è tollerabile, accettabile, soltanto nella miseria e nell'umiliazione. L'uomo nella fortuna, l'uomo seduto sul trono del suo orgoglio, della sua potenza, della sua felicità, l'uomo vestito dei suoi orpelli e della sua insolenza di vincitore, l'uomo seduto sul campidoglio, per usare un'immagine classica, è uno spettacolo ripugnante ${ }^{3}$.

10 Essendo questo un tentativo di comparazione, ciò che giustamente viene subito da chiedersi è di quale natura furono i contatti tra i due. A tal proposito un documento interessante si rintraccia nella nuova biografia di Malaparte scritta da Maurizio Serra nella quale, tra l'altro, viene riportata una lettera che Céline durante il suo periodo in Danimarca inviò a Malaparte per ringraziarlo di una somma di denaro ricevuta; ecco le sue parole nella traduzione italiana fornita da Serra:

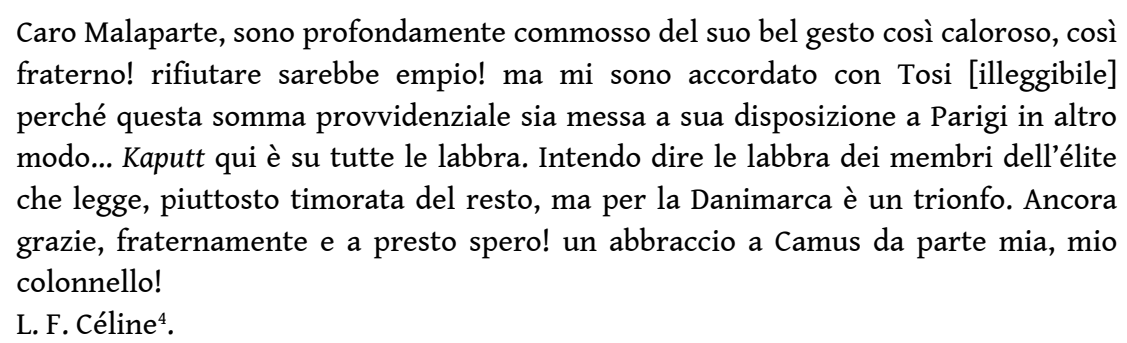

L. F. Céline ${ }^{4}$.

11 Anche se non si può parlare di influenze dirette tra le loro opere o di un'appartenenza a medesime scuole di pensiero, vi fu comunque qualche contatto e non è improbabile che i due si leggessero a vicenda. Il primo ad abbozzare un parallelo tra loro fu Pierre Descaves che aveva parlato di Kaputt come di un libro anomalo in cui «lo stile di Bardamu è rivisto da D'Annunzio» ${ }^{5}$. Un'altra testimonianza di Orfeo Tamburi, pittore amico di Malaparte, ci conferma il fatto che l'autore italiano nutrisse per lo scrittore francese una certa stima:

Malaparte aveva parlato sempre con rispetto dell'uomo Céline, si era intenerito alle sue peripezie e aveva spesso reagito contro l'accanimento che la Francia intera metteva nel disprezzarlo e io so quante volte aveva insistito col suo editore francese [...] affinché ristampasse l'esule che in quel tempo viveva povero e abbandonato in Danimarca.

Conoscevo a memoria la storia, ma Malaparte non si stancava di ripeterla e sempre con successo e con lo stesso entusiasmo.

Ed è vero che gli aveva fatto pervenire le dieci dodicimila corone dei suoi diritti di autore, come era vero che Céline per nulla commosso non gliene era stato affatto riconoscente ${ }^{6}$. 


\section{Il trauma della guerra} traumatica della prima guerra mondiale, nella quale scoprono l'orrore del conflitto, è all'origine delle rispettive opere. La fiducia nei governanti viene presto a mancare e il popolo si rende conto della rottura tra realtà e discorso politico. La guerra provoca disinganno e genera un nuovo atteggiamento di fronte alla morte, come scrisse Freud: «Essa non tiene conto delle limitazioni alle quali ci si attiene in tempo di pace e che formano ciò che chiamiamo il diritto delle genti [...]. Calpesta tutto ciò che trova sul suo cammino, e questo con una rabbia cieca, come se dopo di essa non dovesse esserci avvenire né pace tra gli uomini»?

L'isolamento dell'uomo sentito come tematica filosofica, la povertà esistenziale di stampo nichilista che è consapevolezza della piccolezza dell'uomo di fronte all'universo e al non senso della vita, tematiche al centro di Voyage au bout de la nuit, non hanno invece particolare rilevanza nei romanzi di Malaparte nei quali la negatività della condizione umana risulta piuttosto conseguente a dinamiche di ordine sociale, politico ed economico. È vero che la catastrofe del conflitto ha reso la miseria universale, eliminando confini netti tra ricchi e poveri, vinti e vincitori, ma il suo pessimismo non diviene quasi mai di ordine esistenziale. Scrivo «quasi» poiché echi di un simile nichilismo si ritrovano ad esempio nei suoi romanzi di età adulta e, come scrive G. Grana, «in Kaputt se ne possono anche notare tracce non irrilevanti, congiunte con un opposto senso individuale e anarchico della guerra "rifiutata", alla maniera di Céline $[\ldots] \|^{8}$.

Curioso notare che in entrambi la notevole misantropia e la sfiducia nei confronti del genere umano (aspetti evidenti in particolar modo in Céline) si oppongono all'amore e all'estrema cura che ebbero per gli animali, specialmente per i cani, a cui durante tutta la loro vita dedicarono un'attenzione unica (basti pensare alle immagini di Céline a Meudon); amore di cui, per altro, ci regalano delle bellissime pagine. Sullo sfondo della crudele esibizione dell'uomo, la loro sensibilità li ha portati a vedere e a descrivere con commozione la 'bellezza' della morte di un cane'

Pittori e interpreti della decadenza dell'Europa, i due romanzieri si fanno dunque testimoni di un mondo in cui valori ed ideologie sono del tutto crollati ${ }^{10}$. Le loro opere, tutt'altro che resoconti realisti, danno una visione allucinata della storia; la lotta armata ha svelato un mondo crudele che viene indagato in tutta la sua brutalità e reso attraverso una deformazione grottesca. Si ha una distorsione del reale che rende la storia un ciclo di avvenimenti senza ragione e senza fine. Tale rifiuto in Céline non si traduce però mai nell'intellettualismo lirico e nell'estetismo che invece ritroveremo nelle opere di Malaparte.

Guerra vuol dire disumanizzazione totale, rumore incessante, sfruttamento, oppressione dei deboli, tutti aspetti che torneranno poi nella descrizione dell'alienazione industriale. Ferito da tale dramma, il protagonista di Voyage au bout de la nuit, Bardamu, non può vedere nel mondo che bassezza e oscurità. Egli, anarchico e antipatriota, preso da uno sbalzo di entusiasmo, si lascia trasportare verso l'orrore e ne rimane incastrato «comme un rat»; come i fanti di Malaparte a Caporetto, prende poco a poco coscienza di esser stato usato come pedina del potere. Bardamu è quindi una delle marionette manovrate da qualcosa di grande, tutto gli appare incomprensibile, 
frutto di un errore immenso, una «universelle moquerie» nella quale sente tragicamente la propria inutilità. I burattinai della lotta armata sono i padroni, indifferenti alla morte e alle fatiche di chi combatte. Il rifiuto della guerra nei loro romanzi va quindi di pari passo con un senso di distacco dalla Patria. Come per Barbusse, che in Le Feu scriveva: «Combien de crimes dont ils ont fait des vertus, en les appelant nationales» ${ }^{11}$, anche per i protagonisti del Voyage l'idea di patria e di razza non esistono. Bardamu ironizza sugli ideali patriottici, non crede nella grande Francia, l'eroismo per lui non è che follia:

Serais-je donc le seul lâche sur la terre ? pensais-je. Et avec quel effroi ! ... Perdu parmi deux millions de fous héroïques et déchaînés et armés jusq'aux cheveux? Avec casques, sans casques, sans cheveux, sur motos, hurlants, en autos, sifflants, tirailleurs, comploteurs, volants, à genoux, creusant, se défilant, caracolant dans les sentiers, pétaradant, enfermés sur la terre, comme dans un cabanon, pour y tout détruire $[. . .]^{12}$.

17 La convinzione che il popolo si sia lasciato ingannare dai filosofi, la sfiducia nei confronti delle grandi idee degli intellettuali e dei politicanti, quelli che in Italia barbara Malaparte chiamava $\mathrm{i}$ «falsi sofismi», torna in entrambi gli autori. La decisa ideologia nazionalista che permea le prime opere di Malaparte non gli impedisce infatti di giudicare colpevole l'Italia dei potenti, «matrigna incurante», «mamma marcia», guidata da individui vili ed egoisti di cui i soldati sono vittime indifese. Per Malaparte, allora, di fronte a un potere che continua a considerare la guerra come atto eroico senza però parteciparvi, la rivolta dei fanti diviene inevitabile; alle caste di potere che agiscono come burattinai, lo scrittore riserva i toni più pungenti, traducendo stilisticamente, con immagini crude dal sarcastico gusto rabelesiano tanto amato da Céline, la bassezza del loro valore:

[...] la nazione tutta (o quasi), presa da una fra quelle convulsioni epilettiche che in certi momenti storici si chiamano patriottiche, strillava nelle piazze delle città, sotto ai palazzi degli antichi comuni e intorno ai marmi dagli occhi freddi, mentre gli oratori, dall'alto di scalinate, come Gargantua dalla torre di Nostra-Signora, spandevano l'acido urico delle loro improvvisazioni sulle folle in delirio ${ }^{13}$.

18 Si potrebbe dunque dire che lo spirito che animava il giovane Suckert era di matrice eroica più che patriottica. Alla patria, «véritable raison d'être», «vérité du cœur» ${ }^{14}$, queste le parole del comandante in Voyage alle quali Bardamu non riusce a piegarsi, anche Malaparte ha tolto ogni fiducia: «se tradimento c'è stato, chi ha tradito? I fanti che si sono orrendamente ribellati, o coloro che li hanno spinti alla disperazione?» ${ }^{15}$. Ma attenzione, se nell'animo del soldato di Céline non è auspicabile nessun cambiamento, nessuna consapevolezza maturata, ma regna semplice meccanicismo e spirito di obbedienza nonostante l'assurdità della condizione, Malaparte ci testimonia invece di una graduale presa di posizione dei soldati che, rendendosi conto di essere stati trattati come piccole pedine, vogliono insorgere. Sul piano dell'ideologia politica, non bisogna infatti mai dimenticare che Céline resta sempre un nichilista mentre Malaparte, se non altro per la sua gioventù repubblicana e per la prossima adesione al fascismo ${ }^{16}$, ha una visione progressista della storia; non fu un conservatore che voleva bloccare le sorti italiane in un polveroso immobilismo ma, al contrario, fu fautore di un pensiero progressivo. La ribellione, allora, non è né fuga né tradimento, ma una motivata disobbedienza dei fanti nonché, per lui, possibilità di ritorno alla vera umanità grazie al legame con il popolo degli umili; essa diviene possibilità di incontro con la morte e con la vita, con la paura e l'immensità dell'esistenza. Solo di fronte alla notte «vergine eterna» ${ }^{17}$, gli uomini vivono l'orrore ritrovando attraverso il mistero 
non umano la propria dimensione totalmente umana. Anche il loro diviene un viaggio in fondo alla notte, quella notte che, superfluo dirlo, assume un'importanza simbolica fondamentale anche nel Voyage: «La nuit, dont on avait eu si peur dans les premiers temps, en devenait par comparaison assez douce. Nous finissions par l'attendre, la désirer, la nuit» ${ }^{18}$. La notte paurosa ma dolce di Céline potrebbe così corrispondere all'«infinito inumano» di Malaparte. Inoltre, il «sole cieco» evocato dallo scrittore italiano, metafora di un destino totalmente casuale, è punto cardine della riflessione céliniana. Nel Sole è cieco, quella di cui Malaparte ci parla è una "guerra senza speranza sotto il sole indifferente e impassibile alle sofferenze umane» ${ }^{19}$ : quello del romanzo è un sole che corrisponde alla concezione tragica greca di un destino indifferente alle sorti umane: elemento di fondamentale importanza anche nella concezione esistenziale di Céline. Come nel Voyage, gli uomini in guerra sono in lotta tra di loro senza comprenderne le ragioni profonde, partecipano a un gioco insensato senza conoscerne le regole. La morte, questo è un punto di comunione che mi pare centrale nei due, è meccanica e spietata, essa arriva e caccia via questi «uomini piccoli e grigi» che vedono la guerra come una fatalità inevitabile e che vi sono entrati senza odio, contro la loro volontà ma con l'impegno dei lavoratori «schiacciati dalla presenza continua della morte, dalla presenza insofferibile di una forza che rendeva la loro vita non necessaria» ${ }^{20}$.

In Voyage au bout de la nuit, come già accennato, si può scorgere un influsso dell'autore francese Henri Barbusse, uno dei pochi narratori di cui Céline ammise una certa influenza («Pour mon petit personnel je dois beaucoup à Barbusse») ${ }^{21}$, per la forte condanna della guerra e per l'utilizzo del linguaggio parlato da parte dei soldati. Come per Malaparte e Céline, anche per Barbusse, partito come volontario per il fronte, l'esperienza della trincea costituisce un trauma, un'epifania che si trasforma in scrittura. Nel suo romanzo Le Feu, prima opera che si impone nel panorama letterario per la cruda descrizione della vita e delle sofferenze dei soldati francesi, proponendo una testimonianza realistica della brutale esperienza della guerra, egli esprime il rifiuto del militarismo e di qualsiasi retorica nazionalista ed eroica. Riflessioni come quelle meditate in Le Feu: «Je reste dans le noir et le silence [...] je ne sais pas ce que je suis, où je vais, ce que je fais, mais moi aussi j'ai crié du fond de mon âme vers un peu de lumière ${ }^{22}$, potrebbero essere concepite dal protagonista del Voyage.

Il libro di Barbusse aveva, inoltre, la stessa tesi centrale della Rivolta: la guerra non era stata voluta da chi la combatteva ma giovava a chi l'aveva voluta. Viva Caporetto!, per altro, nel quarto ed ultimo numero della rivista Oceanica, di cui Malaparte fu ideatore e direttore, venne proprio sponsorizzato come libro d'attualità di un «Barbusse italiano». Ci si sente dunque di concordare con l'opinione del critico Giordano Bruno Guerri, quando scrive che la Rivolta dei santi maledetti si rifà a quell'Henri Barbusse in qualche modo 'ispiratore' dell'oceanismo ${ }^{23}$, movimento culturale, artistico e filosofico di cui Malaparte fu creatore, elegante appello contro la borghesia, la scienza ed il progresso a favore del recupero dell' infinito e del tragico, del senso di umanità e passione che erano ormai perdute.

\section{La visione della miseria}

Altro punto in comune tra gli autori risiede nelle atmosfere completamente cupe, quasi oniriche, in cui tutto si trova avvolto nel buio, nell'ombra, nell'odore di polvere, nel 
fragore continuo. Ciò che ritroviamo è quindi il coraggio di immergersi nelle fogne dell'umanità, di sguazzare tra i miserabili condannati alla sofferenza e inclini al peccato, il tutto reso da una narrazione piena di emozione, di fantastico, di atmosfere crude, di drammatici contenuti in cui il primordiale è elemento fondante. Sicuramente la scrittura céliniana è più caotica e ha una ritmica più incalzante ma ciò che torna è il rimpianto per un mondo non dominato dal progresso e dalla ragione, in cui ci sia maggior bellezza, spontaneità e grazia, in cui il naturale e l'istintivo siano al di sopra di ogni sottomissione. Céline lo fa squartando la lingua razionale e chiara, Malaparte lo esprime attraverso una scrittura lirica. Una condizione di nausea permanente rende $\mathrm{i}$ personaggi simili a bestie e questa animalità è un aspetto molto importante della comparazione; in Malaparte essa prende spesso il significato di innocenza, ma in entrambi la metafora animale significa soprattutto rabbia, violenza, dolore: «Allemagne, France et Continents, tout ce qui respire, détruire, plus enragés que les chiens, adorant leur rage (ce que les chiens ne font pas), cent mille fois plus enragés que mille chiens et tellement plus vicieux! Nous étions jolis! Décidément, je le concevais, je m'étais embarqué dans une croisade apocalyptique ${ }^{24}$ e Malaparte nella Pelle: «Una folla smunta e lacera seguiva il corteo piangendo e imprecando: e le donne si strappavano i capelli, si ficcavano le unghie nel viso e, denudandosi il petto, alzavano gli occhi al cielo, ululando come cagne» ${ }^{25}$.

Seppure in entrambi i casi i soldati siano animalizzati e descritti come vittime incoscienti di un sistema che li sovrasta del tutto indifferente alla loro sorte, i poveri protagonisti di Malaparte sono spinti da un istinto che li porta a esser buoni con chi soffre, ed è questo sentimento di solidarietà, che commuove Malaparte, a rappresentare l'elemento più dissimile tra $\mathrm{i}$ due autori analizzati. Antitetico all'uomo povero $\mathrm{e}$ selvaggio sta il borghese che continua a svuotarsi di ogni aspetto umile per adagiarsi in un conformismo alienante; ma se in Malaparte si sente l'eco del mito rousseauiano dell'uomo selvaggio, secondo il quale la bontà è propria solo all'uomo naturale che conserva un'anima intatta e genuina e che può ancora gioire della pura esistenza, in Céline la bestializzazione perde i caratteri di genuinità e ingenuità; il povero di Céline, in quanto uomo più istintivo, è quasi sempre spontaneamente sottomesso alle autorità e mosso da un egoismo cieco e dunque il pensiero céliniano, al contrario di quello di Malaparte, molto difficilmente potrà definirsi populista ${ }^{26}$. Certo, la sua attenzione alle condizioni sociali e mediche delle classi meno abbienti e all'ingiustizia del sistema, torna in innumerevoli casi e ogni volta con sfumature diverse, ma in lui il popolo non viene mai idealizzato.

Il mondo céliniano è senza speranza, disincantato; andare altrove alla ricerca di verità o di benessere, allora, è solo un'illusione. La pesantezza, che egli riesce a rendere anche attraverso l'artificio stilistico, uniformizza il mondo rendendo impossibile ogni evasione. Un cambiamento sociale, nella visione cinica di Céline, è difficilmente auspicabile, tutto sembra avvolto da una piattezza e da un'immobilità che risultano antirivoluzionarie. Céline ribalta quindi il mito del buon selvaggio di Rousseau, ne fa una parodia: «on peut en effet lire Voyage au bout de la nuit comme des Confessions à l'envers ${ }^{27}$. Alla generosità oppone la crudeltà, alla purezza la cattiva coscienza. Nel romanzo, così come sarà nel suo primo libello Mea culpa, è dunque sviluppata una concezione che non crede nella bontà innata dell'uomo, una filosofia divergente con la tradizione libertaria. Sono rarissimi i casi in cui Céline lascia uno spiraglio di speranza e finalmente ci descrive dei personaggi che, nonostante tutto, mantengono una certa 
bontà. Un personaggio che incarna la generosità, ad esempio, è il sergente Alcide che si sacrifica per la nipote orfana decidendo di prolungare il suo impegno nelle colonie; nonostante la sua rassegnazione, la sua umiltà lo rende uno dei pochi personaggi positivi agli occhi di Bardamu:

C'était d'ailleurs une bonne nature Alcide, serviable et généreuse et tout. Je le compris plus tard, un peu trop tard. Sa formidable résignation l'accablait, cette qualité de base qui rend les pauvres gens de l'armée ou d'ailleurs aussi faciles à tuer qu'à faire vivre. Jamais, ou presque, ils ne demandent le pourquoi les petits, de tout ce qu'ils supportent. Ils se haïssent les uns les autres, ça suffit ${ }^{28}$.

Altra eccezione è Bebert, personaggio che infatti ci può ricordare gli ingenui e buoni soldati del Sole è cieco di Malaparte: «Sur sa face livide dansotait cet infini petit sourire d'affection pure que je n'ai jamais pu oublier. Une gaieté pour l'univers» ${ }^{29}$. Nonostante questa chiara differenza, nei due torna la descrizione del dramma di chi è vittima inerme della società. Céline conosce e vuole parlare dalla prospettiva della miseria, dello squallore della periferia, dell'abbrutimento sociale di gente senza futuro e senza fede. Lo fa ispirandosi ai paesaggi della sua fanciullezza e ai luoghi della periferia parigina in cui lavorerà da medico. L'autore presenta scenografie, immagini e storie di degrado morale e fisico, leggendo le sue pagine bisogna far l'abitudine ad ambienti sporchi e poveri, si entra nei meandri di una società abitata da reietti, in case turpi tra uomini che vivono in stato di indigenza, sia che si tratti delle banlieues parigine che di altri luoghi da lui indagati altrove. È soprattutto in questo senso che si può parlare di uno scrittore 'vicino' al popolo. La vicinanza va intesa in primo luogo come antiborghesia: i borghesi, ed è questo uno dei punti che mi pare avvicinare maggiormente i due narratori qui analizzati, sono nella loro ottica i carnefici della polverizzazione della società e dei valori, vuoti automi adoratori del dio denaro. La borghesia rappresenta il decadimento degli ideali, è dunque descritta come moralmente sporca e corrotta.

Come Céline, anche Malaparte, nonostante appartenesse a un mondo diverso, durante la sua vita cercò sempre di mescolarsi al popolo, quel popolo che, dopo aver sostenuto il peso della lotta, viene respinto dai vecchi ceti dirigenti e utilizzato per i giochi di potere; egli cerca di comprenderlo, di guardarlo da vicino: «Il mio continuo sforzo di comprendere, di mescolarmi al popolo, di giudicare da vicino la vita delle masse, e quella istintiva tendenza a giustificare il mio cinismo, la mia libertà di spirito, fuori dall'ambiente culturale che è il mio, col contatto con un mondo morale che non è quello della mia cultura $»^{30}$.

Le condizioni di vita e di lavoro del proletariato non lasceranno indifferenti i due e Malaparte, anche negli anni in cui lavorò a Torino come direttore della Stampa, accettava con difficoltà le scelte della Fiat e provava a mischiarsi agli operai per farsi interprete dei loro bisogni. A contatto con gli operai: «Era il suo cuore garibaldino e d'uomo di sinistra fascista che ricominciava a battere, nonostante l'assuefazione ai salotti e ai dividendi dei consigli di amministrazione» ${ }^{31}$.

Entrambi sviluppano dunque l'idea secondo la quale l'egoismo dell'uomo è potenziato dagli effetti corruttori della società, parlano dell'umanità civilizzata con disgusto, deplorano l'incostanza del mondo moderno e descrivono l'uomo del popolo come uomo presociale, sotto-umano, bestiale. L'impotenza dell'uomo moderno è data dall'incapacità di dominio in un mondo sempre più brutalizzato dalla società capitalistica. Giocando un ruolo nella collettività l'uomo perde la sua essenza e si riduce alla sua apparenza; questa contraddizione insita nella società crea nell'uomo una 
disarmonia. La scrittura deve essere tentativo di andare contro corrente, la critica alla contemporaneità si impone in nome della libertà di espressione.

\section{Parigi e Napoli: città volgari e decomposte} storico del secolo scorso in cui Malaparte, nei panni dell'uomo di strada e del vinto, si fa interprete della decadenza dell'Europa post-bellica. La guerra, questa volta insieme alla peste, risulta responsabile del sacrificio e delle sofferenze, è causa di corruzione.

La «pourriture» ossessiva di Voyage au bout de la nuit torna con la stessa insistenza nel romanzo di Malaparte. Tutto è pervaso dal marciume:

Eppure, tutto ciò che quei magnifici soldati toccavano, subito si corrompeva. Gli infelici abitanti dei paesi liberati, non appena stringevano la mano ai loro liberatori, cominciavano a marcire e a puzzare. Bastava che un soldato alleato si sporgesse dalla sua jeep per sorridere a una donna, per accarezzarle fugacemente il viso, perché quella donna, serbatasi fino a quel momento dignitosa e pura, si cambiasse in una prostituta. Bastava che un bambino si mettesse in bocca una caramella offertagli da un soldato americano, perché la sua anima innocente si corrompesse ${ }^{32}$.

La potenza allucinata di deformazione del reale dà una forza particolare sia alla scrittura di Malaparte che a quella di Céline, «brueghelien par istinct» ${ }^{33}$; è una scrittura in cui miseria e follia sono dappertutto come in un quadro del pittore fiammingo. Nulla di romantico permane, non c'è consolazione ma costante precarietà e i luoghi sono dunque oscuri e infernali. A tal proposito interessante che l'allegoria della mostruosità, questa torbida fantasia grottesca, porti entrambi ad avere Bosch, Dürer, Brueghel, Goya come fonti di ispirazione dei loro romanzi:

Nane calve e sdentate vanno su e giù per i viscidi scalini, appoggiandosi a bastoni, a grucce, traballando sulle gambine corte, alzando il ginocchio fino al mento per salire sul gradino, o si trascinano carponi, mugolando e sbavando: paiono mostriciattoli di Brueghel o di Bosch, e un giorno una ne vedemmo, Jack ed io, seduta sulla soglia di un antro con un cane malato in braccio. In quel grembo, fra quelle braccine, il cane pareva un gigantesco animale, una mostruosa belva ${ }^{34}$.

E Céline aveva altresì assimilato la propria scrittura alla pittura di Brueghel:

Vous connaissez certainement, Maître, l'énorme fête de fous de P. Brueghel. Cela est à Vienne. Tout le problème n'est pas ailleurs pour moi [...]. Tout mon délire est dans ce sens et je n'ai guère d'autres délires. Je ne me réjouis que dans le grotesque aux confins de la mort. Tout le reste m'est vain ${ }^{35}$.

Neanche Malaparte cerca una narrazione oggettiva. Il suo viaggio de La pelle è un viaggio al termine della notte attraverso la penisola italiana, viaggio estremamente più breve di quello céliniano e che si compie quasi unicamente nella città di Napoli. La nausea céliniana dell'uomo annichilito si legge tra le pagine di questo romanzo soprattutto nelle descrizioni volutamente ridondanti del popolo sofferente di Napoli. Inoltre, come nota Maurizio Serra, si ha la percezione della fine dell'Europa a cavallo della Seconda guerra mondiale presente, tra l'altro, nella «saga della fuga attraverso la Germania in fiamme di Céline» ${ }^{36}$.

33 Napoli, come lo erano la Parigi e la New York di Céline, è una città corrotta in ogni suo aspetto, volgare e decomposta, seppur tale decomposizione si presenti in uno scenario e con motivazioni differenti: il caos e il robotismo delle città di Céline causati dal capitalismo, si trasformano qui in un disfacimento che è invece segno della sconfitta e 
della fine della civiltà europea. Tutto al suo interno è degradato e privo di dignità, ma nonostante l'ambiente e le azioni del popolo siano così deteriorati e depravati, i napoletani, così contraddittori, riescono ancora a soffrire e a piangere per gli altri e «il dolore di ciascuno è il dolore di tutta la città» ${ }^{37}$. Malaparte, come Céline, descrive ambienti sudici, turpi, tuguri e luoghi di estrema povertà senza lasciarsi prendere da alcun sentimentalismo. Tutto nella Napoli de La pelle è in vendita, ogni cosa vi si commercia in maniera ignobile, che siano bambini o il rapporto con una vergine. Gli uomini sono mossi da paura e da disprezzo céliniano, l'«universelle moquerie» del Voyage diviene nel romanzo malapartiano «universale vergogna»:

Bisogna riconoscere che facevano di tutto per essere indegni del nome di uomini, pochissimi erano coloro che si serbavano intatti, come se il morbo nulla potesse contro la loro coscienza: e si aggiravano timidi, spauriti, disprezzati da tutti, quali importuni testimoni dell'universale vergogna ${ }^{38}$.

Malaparte, in un certo senso, sembra giustificare gli atti dei napoletani poiché causati da una reale sofferenza, «Si les gens sont si méchants, c'est peut-être parce que qu'ils souffrent ${ }^{39}$ diceva Bardamu nel Voyage. A Napoli, come nella Parigi di Céline, soprattutto quella di Mort à crédit, la gente ha smesso di guardarsi veramente e, sospettosa, si spia.

Ma, nonostante questa insistenza nell'immersione di ambienti deteriorati e putrefatti dove l'egoismo è al di sopra di tutto, comune all'autore francese, c'è un aspetto predominante in Malaparte che lo distingue profondamente dalla visione céliniana e cioè la sua visuale moralistica di stampo cristiano che insiste sulla pietà. Tale aspetto viene particolarmente ribadito nel capitolo dedicato alla "vergine di Napoli», dura testimonianza dell'umiliazione cui erano giunte molte donne a causa della disperazione. Napoli è descritta come un «abisso di vergogna e di dolore, un inferno di abbiezione $»^{40}$, la degradazione delle donne e dei bambini, però, non è qui delineata come un vizio, ma è piuttosto la manifestazione della corruzione causata dalla guerra $\mathrm{e}$ dall'ambiente: «la sua volgarità non aveva nulla di personale. Pareva piuttosto un riflesso della volgarità dell'ambiente» ${ }^{41}$, scrive Malaparte a proposito della giovane donna costretta ad esibire la propria verginità. Egli non sembra riuscire a proporre una soluzione, pare quasi compiacersi dei mali che descrive sempre con veemenza e suggestione violenta. Ci introduce in una città infernale in cui vero $\mathrm{e}$ falso, bene e male si confondono. «Ça serait pourtant pas si bête s'il y avait quelque chose pour distinguer les bons des méchants» scriveva Céline nel Voyage. Lo stesso dilemma ricompare nelle pagine di Malaparte:

Nella Pelle la guerra non è conclusa, ma la sua fine è già stata decisa. Le bombe continuano a cadere, ma questa volta cadono su un'altra Europa. Ieri non ci si chiedeva chi fosse il boia e chi la vittima. Ora, improvvisamente, il bene e il male hanno il volto velato; il nuovo mondo è ancora poco conosciuto; ignoto; enigmatico; colui che racconta ha una sola certezza: è sicuro di non essere sicuro di nulla ${ }^{42}$.

Altro punto essenziale della loro riflessione sulla decadenza del mondo occidentale e della nullità dell'uomo contemporaneo è legata all'anonimato delle città. New York in Voyage au bout de la nuit e Londra in Mort à crédit rappresentano l'uomo perso in una folla che lo inghiotte, l'individuo stesso ridotto a numero e capitale:

Ils avançaient les gens vers la lumière suspendues dans la nuit au loin, serpents agités et multicolores. De toutes les rues d'alentour ils affluaient. Ça faisait bien des dollars, pensais-je, une foule comme ça, rien qu'en mouchoirs, par exemple, ou en bas de soie! Et même rien qu'en cigarettes! Et dire que soi-même, on peut se promener au milieu de tout cet argent, ça ne vous en donne pas un seul sou en plus, 
même pour aller manger! C'est désespérant quand on y pense, combien c'est

défendu les hommes les uns contre les autres, comme autant de maisons ${ }^{43}$. il protagonista, Ferdinand, è immerso in una folla rumorosa mossa da cieca curiosità e in cui tutto è menzogna. Tale folla resa sporca dalla corruzione della democrazia e dalla contaminazione dei costumi è presente anche in Diario di uno straniero a Parigi di Malaparte:

È la folla delle sei di sera, dei piccoli borghesi dei commessi di negozio, delle dattilografe, l'orribile folla piccolo borghese delle sei di sera in ogni città di Europa, sporca, stanca, vestita con pretensione, affaccendata, egoista, astiosa, cattiva, fredda, sovrana. [...] Odio questa folla che vi urta, vi calpesta, vi schiaccia ${ }^{44}$.

\section{Una scrittura grottesca e visionaria}

Nella loro scrittura lo spettacolo della misera condizione umana, della sua infelice bassezza è però sempre accompagnato da un'ironia di fondo, da un gusto per il grottesco e per il riso, da una narrazione ricca di satira e di humour, come se nell'oscurità e nel delirio permanesse sempre una certa logica. Gli autori descrivono quindi un clima fatto di sporcizia, grida, pianti, rumori, violenza ma sempre in uno sfondo comico, o meglio, tragicomico.

Se da un punto di vista tematico sono molti i rimandi, lo stesso non può esser detto però del loro stile; per quanto in entrambi ci sia un'attenzione particolare alla forma $\mathrm{e}$ una volontà di scrittura unica ed elaborata, le loro scelte linguistiche sono estremamente diverse.

Céline vuole andar oltre lo stile ufficiale, sorpassare i raffinati della cultura. Nei suoi romanzi c'è un'esplicita volontà di andare al di là dei codici prestabiliti, egli trasmette una sorta di innocenza astiosa. Se la lingua ufficiale lavora al mantenimento dell'ordine, l'arte e la poesia devono cercare forme diverse per distinguersi da essa e lavorare, al contrario, alla sovversione dell'ordine stesso. In Entretiens avec le Professeur $Y$, l'autore insiste sulla sua capacità di trasporre la vita autentica, come se il solo modo di scappare al robotismo fosse il ritorno al proprio ritmo emotivo. L'autore vuole rigettare il linguaggio inteso come puro ornamento stilistico e rendere l'immersione nel mondo descritto.

41 La sua scrittura si situa allora in opposizione al classicismo, così come in Italia succede, ad esempio, con Carlo Emilio Gadda, simile a Céline per un certo gusto che va di pari passo con un'ideologia individualista che si vuole in rottura col passato, seppur il plurilinguismo gaddiano, come ha ben notato Ernesto Ferrero ${ }^{45}$, sia più duttile e mobile. Contini ci propone tale analisi:

Più vicine alle nostre letture quotidiane, la produzione francese, tra le innumerevoli con cui reagisce al suo disseccamento cartesiano [...] annovera pure le operazioni espressionistiche, all'ingrosso gergali, di Céline (con cui certo Michaux) e di Audiberti $[. . .]^{46}$.

Solo utilizzando frasi sintatticamente scardinate, neologismi, ripetizioni, un lessico basso e spesso volgare, il mondo dei subalterni può aver voce. Stile e ideologia sono intimamente interconnessi. Tutto nelle descrizioni è pervaso da un'atmosfera di terrore e desolazione, Malaparte grida la sua collera con «turpitudine céliniana»" il suo stile, piuttosto barocco e retorico, può risultare insistente nella volontà di 
ridondanza e di furore polemico. Aspetto, questo, che per il critico Gianni Grana rimanda al «peggior Céline»:

La volgarità non è tanto nelle cose, e non è nemmeno nelle invenzioni in se stesse, quasi sempre di efficace concezione, come necessario linguaggio della verità assurda, della crudeltà dolorosa, di una Napoli-Europa ridotta alla pelle, nel suo pietoso inferno; ma è piuttosto nel gusto deteriore della pagina, in una qualità descrittiva rudimentale e retorica, nelle insufficienze e approssimazioni tecniche e stilistiche di un libro enfatico e disformato, che talvolta fa pensare al peggior Céline, se non altro per la mancanza di gusto e il furore polemico ${ }^{48}$.

Come in Céline la tragedia e l'ironia vanno di pari passo: «È un mondo colto nel suo realismo minuto, senza giudizi preconcetti, in cui la farsa va di pari passo con la tragedia e il dolore non è quasi mai assunto come tale, bensì convertito in beffa $\mathrm{e}$ irrisione ${ }^{49}$. Il riso è quindi un aspetto che unisce i due, punto essenziale della scrittura come critica sociale:

Il ridere fa male al cuore degli ipocriti, il riso è un'opinione. Il pianto, no, il pianto non è che un sentimento ... il riso è una condanna. È il riso che ha governato la libertà di Atene, di Firenze, di Parigi. Soltanto nei paesi senza libertà, Roma, Sparta, Berlino, il riso è considerato un atto sedizioso. Quando i popoli di Europa avranno imparato nuovamente a ridere, a ridere di tutti, specie di chi comanda, l'Europa sarà salva ${ }^{50}$.

Sono la scrittura impregnata di commozione, la costante ricerca di equilibrio nello stile, il linguaggio compiaciuto e intellettuale a distinguerlo invece dallo stile céliniano. Inoltre, anche quando egli più si avvicina al linguaggio parlato con un'evidente volontà di mimesi, lo fa sempre mantenendo una fusione con i moduli tradizionali. Per esempio, si ha questa tendenza al verismo nel Sole è cieco in cui egli utilizza delle espressioni dialettali attraverso una sintassi sperimentale piena di anacoluti e di discorsi indiretti liberi; ma la sua sperimentazione linguistica rappresenta piuttosto una composizione che unisce le forme dialettali a una ricerca di poeticità. È invece il gusto per lo stravagante in cui l'inverosimile si colora di grottesco ad accomunarli. La loro vuole essere una scrittura piena di eventi bizzarri.

Del classicismo Malaparte conserva l'evocazione di un destino indifferente alle sorti umane e il culto tragico della morte, morte dell'anima e della dignità: «che cosa conta l'anima, ormai? Non c'è che la pelle che conta» ${ }^{51}$, sono parole che possono rievocare quelle di Bardamu: «les élans du cœur m'étaient devenus tout à fait désagréables. Je préférais ceux du corps, tout simplement. Il faut s'en méfier énormément du cœur, on me l'avait appris et comment ! à la guerre» ${ }^{52}$.

I due narratori rievocarono quindi quello che videro in prima persona, ma il contatto tra la realtà vissuta e il racconto è ridotto al minimo: «il suo era uno Zola dimezzato, gravemente mutilato» ${ }^{53}$ scrive Bo di Céline. Anche se Céline stesso in una lettera a Élie Faure si distingue proprio da Malaparte: «Malaparte [...] il accumule les horreurs ? La belle histoire! C'est rien ça. C'est les faire chanter qui est fortiche. Une chanson de Bruant m'en dit plus long que trente kilos d'Eugene Sue» ${ }^{54}$. Sebbene Céline in questa lettera parli della scrittura malapartiana come «accumulo di orrori», con un'esplicita volontà di distinguersi grazie alla sua capacità di trasformarli in canto, in realtà anche in Malaparte si ha la volontà di posare sulla realtà uno sguardo che vada oltre la neutralità dipingendo gli orrori dal dentro $\mathrm{o}^{55}$, trasformando le atrocità in arte. Vero $\mathrm{e}$ fantasia si confondono nei loro romanzi e diviene inutile cercare di separare ciò che è vero da ciò che non lo è. Interessante che in entrambi torni la rivendicazione della 
libertà artistica attraverso un parallelismo con l'opera pittorica. Malaparte scrive a tal proposito:

L'écrivain, devant les horreurs de la guerre, pourquoi devrait-il se contenir d'une façon différente que le peintre? Les capricios de la guerre, de Goya, les hallucinantes gravures de Callot, sont-ils un fruit de la fantaisie de ces peintres, ou la réalité est telle que la voit un oeil objectif ? On réclame de l'écrivain, du peintre, que leur cœur commande à leurs yeux. C'est le contraire qui est vrai. L'œil est un organe indépendant du cœur. Si l'artiste s'émeut, il ne voit plus ${ }^{56}$.

E Céline, non discostandovisi molto, in Entretiens avec le pofesseur $Y$ rivolgendosi all'immaginario giornalista che lo sta intervistando dice:

Vous reprochez pas à Van Gogh que ses églises soient biscornues? À Vlaminck ses chaumières foutues !... à Bosch ses trucs sans queues ni tête ?... à Debussy de se foutre des mesures? Honegger de même! Moi j'ai pas du tout les mêmes droits? non? J'ai que le droit d'observer des Règles ?... les instances de l'Académie ?... c'est révoltant ! ${ }^{57}$

Si susseguono quindi storie vissute e proiezioni immaginarie, il reale si trasforma in onirico, in atmosfere visionarie e inquiete.

Da Viva Caporetto! ai romanzi in Malaparte si ripete uno stile incalzante carico di immagini e parole, retorico e tendente all'enfasi; riappare il gusto, lampante in Céline, dello scandalo, dell'invettiva e della provocazione, che sembra volere esprimere la loro profonda necessità di scrivere liberamente e di dire quello che gli altri non hanno il coraggio di esprimere.

\section{La reinvenzione del sé}

50 Altro punto fondamentale di similitudine tra i due è la sistematica riscrittura che fecero della loro personalità attraverso i loro romanzi; certamente i loro scritti hanno un carattere autobiografico, ma è un'auto-narrazione in cui il vero e il falso si confondono. Nella loro opera, come nella vita, vollero lasciare un'immagine sempre sfuggente e incerta. A tal proposito su Céline è stato scritto:

On admet aujourd'hui, non seulement, l'évidence d'une affabulation progressive chez l'auteur, mais l'identification croissante de Louis-Ferdinand à Bardamu et Ferdinand, jusqu'à ce que l'auteur et son double ne fassent plus qu'une seule et même personne, Céline parvenant à la fin, à faire coïncider son monde avec le monde : en effet, tout se passe comme si l'auteur, dès la publication du Voyage, avait renchéri sur la presse, abusée ou provoquée, comme s'il avait conspiré à fausser son image, celle-là même qui, plus tard, ne manquerait pas de lui nuire $[. . .]^{58}$.

51 Un aspetto di capitale importanza è quindi la reinvenzione del sé attraverso la letteratura. Essi furono uomini egocentrici, affabulatori, dal percorso di vita caotico e romanzesco.

52 Anche la loro concezione del potere, e in particolare delle dittature e il rapporto che instaurarono con esse, fu tutt'altro che lineare, il loro percorso politico e di vita fu infatti burrascoso e travagliato. I due scrittori furono due individualisti brillanti e cinici. Diviene difficile quindi distinguere l'uomo dal personaggio, come se vita e opera si confondessero; i loro scritti divengono parte della loro vita creando la leggenda della loro storia personale.

È comprensibile come le scelte di Malaparte possano esser lette come opportuniste e lui come 'voltagabbana', la sua pretesa dannunziana di far della sua vita un'opera d'arte fa 
però da sfondo ad una reale esigenza di libertà che sovrasta le sue scelte contraddittorie: «Le contraddizioni dell'uomo e dello scrittore si spiegano, così, come ambivalenze insolubili di uno spirito disarmonico, drammaticamente conviventi nell'animo" ${ }^{59}$. Se egli ha abbandonato delle cause non è stato per andare dalla parte dei vincitori ma sempre perché era il suo temperamento a guidarlo. $\mathrm{Fu}$ un uomo coraggioso che mai amò seguire il politicamente corretto.

La costatazione del vuoto e della corruzione della società borghese conduce il giovane Malaparte allo sviluppo di un pensiero rivoluzionario che trova espressione compiuta nel movimento squadrista del fascismo degli albori; ma ciò che lo rende uno scrittore atipico è che ben presto la critica intransigente, nonché l'analisi attenta delle dinamiche di potere espressa nei suoi scritti, lo portano ad un distacco da Mussolini che infatti lo allontanerà mandandolo al confino. Il processo delle sue idee sarà ricco $\mathrm{e}$ niente affatto circoscritto all'esperienza fascista.

Contraddittorio certamente lo è anche Céline: se Voyage au bout de la nuit, suo primo romanzo, fu un grido di denuncia e perciò piacque estremamente agli intellettuali di sinistra, anarchici e comunisti, la pubblicazione di Bagatelles pour un massacre, nel 1937, rivelò uno scrittore animato da un antisemitismo viscerale e vicino a posizioni di destra, sconvolgendo perciò la critica letteraria del tempo. Non si può pensare che le sue invettive siano mero artificio retorico, ma si ha come l'impressione che egli voglia sempre effettuare un discorso radicale per gridare contro ipocrisie a favore di un estremismo che vuole essere sinonimo di autenticità. Tuttavia sarebbe superficiale non riconoscere una continuità all'autore e parlare di un doppio Céline, come se non vi fosse un filo rosso a legare il romanziere al pamphletaire fascista e collaborazionista. Il Céline mosso dalla denuncia «della devastante condizione materiale e spirituale in cui è precipitato l'individuo nella società moderna» ${ }^{60}$ intrattiene stretti rapporti teoricopolitici con la scelta successiva dell'antisemitismo. Egli andò sempre contro chi voleva rinchiuderlo in classificazioni schematiche e cercò di star lontano dagli omaggi per restare coerente alla negazione del mondo e alla sua idea di rivolta, contro la borghesia, il denaro, l'esercito, l'ordine, contro la retorica dei sentimenti, le convenzioni banali e le buone maniere. $\mathrm{Fu}$ aggressivo e buono allo stesso tempo, cinico ma malinconico, ironico e provocatore.

Ciò che qui si crede di poter sostenere è quindi un certo anticonformismo dei due autori che, nonostante un apparente paradosso, hanno mantenuto una coerenza nella loro pretesa di verità.

57 Come è ben noto, in Bagatelles pour un massacre Céline esprime l'idea secondo cui gli ebrei hanno invaso ogni aspetto della vita dei francesi e il significato di juif sembra andar oltre il suo nucleo semantico e come una sorta di sineddoche abbracciare tutto ciò che è sinonimo di meccanicismo capitalista. Interessante notare come vi siano dei punti in comune con il Malaparte 'selvaggio', per la tensione polemica continua, la furia espressiva, le tinte forti, estranee poi al Malaparte più maturo. Troviamo dei rimandi all'antisemitismo di Papini e Soffici e alle parole che Maccari scriveva, più di dieci anni prima, nel Selvaggio, rivista vicina a Strapaese di Malaparte ${ }^{61}$. Non c'è però in Céline l'insistenza per il recupero della tradizione che invece, come abbiamo visto, è di fondamentale importanza nel primo Malaparte:

Ciò che fa di Céline un intellettuale tutto immerso nella modernità del Novecento è l'assenza di qualsiasi atteggiamento nostalgico. Al contrario di un Maurras, che sviluppa il proprio pensiero politico sulla contrapposizione fra la Tradizione e la 
Modernità, in Céline la nostalgia della Tradizione evapora del tutto, caratterizzando

il suo antisemitismo come una risposta alla modernità borghese e comunista $[. . .]^{62}$. andata persa sono aspetti che, ancora una volta, possono farci pensare al giovane Malaparte quando in Italia barbara esprimeva l'ansia per la minaccia dell'europeismo che rischiava di sopraffare lo spirito nazionale. Ma in Bagatelles l'omologazione del mondo sotto il peso insopportabile dell'economia di mercato diviene sinonimo di ebraizzazione: «Nous n'avons jamais eu de roi, de président du conseil, de conventionnel, de "chef" qui n'ait été au moins deux ou trois fois vendu à quelque puissance étrangère. C'est-à-dire en définitive à la juiverie» ${ }^{64}$. Ciò che in questa sede è importante ribadire è quindi il filo conduttore che unisce i due e che sta nella volontà di battersi attraverso la forza del loro linguaggio contro la maschera del potere prestabilito, le lobbies, il colonialismo, il militarismo.

Ci si sente dunque di concordare con l'analisi proposta dalla Morand quando rispetto a Céline sostiene:

[...] une analyse d'ensemble de sa pensée politique montre qu'il participe à la fois et en même temps du tempérement de droite et du tempérament de gauche [...] dans le dédale des idéologies politiques il se fourvoya et se vit puni pour s'etre laissé tenter par elles. Il ne sut ni prévoir la portée de ses écrits ni en mesurer les conséquences et il ne lui apparut pas que la responsabilité du penseur et de l'ecrivain puisse être aussi grande que celle de l'homme d'action ${ }^{65}$.

\section{I saggi malapartiani}

62 Come già specificato, considerato che l'aspetto antiborghese di Malaparte, classico nel suo stile, non è così sovversivo come in Céline, il rimando ai saggi politici malapartiani può forse apparire un po' azzardato, ma ciò che mi preme ribadire è l'atipicità del loro 'legame' con le dittature.

Non si può non ammettere il carattere particolare che Malaparte ebbe nell'approccio al fascismo, fu un fascista rivoluzionario ma sempre critico nei confronti del potere mussoliniano che vedeva avanzare; uomo indipendente, anti-disciplinato, si trova accanto al potere e ben presto in conflitto con esso. Come venne scritto in un articolo del 1933, riportato da Serra nella sua biografia dell'autore: «Fascista Malaparte lo era, ma fascista indipendente, troppo ardente e troppo cosciente del suo valore per accettare ciecamente delle direttive» ${ }^{66}$.

Punto che lo distingue nettamente dal 'fascismo' di Céline è il suo distacco totale dalla propaganda antisemita, non c'è razzismo né visione cospirazionista e i toni del suo saggio politico sono del tutto distinti da quelli impetuosi di Bagatelles, seppur anche in lui si celi un pessimismo di fondo e una seria preoccupazione per un futuro che potrebbe rivelarsi catastrofico. Anche per Malaparte l'avvenire è minaccioso. Se Céline 
aveva parlato di follia per descrivere il seguito hitleriano ma, allo stesso tempo, non smentendo mai le sue contraddizioni, si era mostrato estremamente vicino all'ideologia antisemita del Führer, Malaparte è invece estremamente critico.

Nei suoi saggi politici ci offre dunque un'analisi oggettiva e complessa, del tutto estranea all'invettiva di Bagatelles e alla retorica dei suoi primi scritti saggistici. Come giustamente nota Maurizio Serra: «non troviamo in Malaparte le invettive deliranti e la xenofobia di un Céline, la paura del contagio e dell'usura di Pound, la venerazione di un Maurras per il trono e per l'altare $\aleph^{67}$.

Seppur la differenza con i libelli di Céline sia indiscutibile, certe parole di Malaparte ci offrono un rimando interessante all'autore francese per la comune volontà di distinguersi dall'egoismo degli uomini, auto-rappresentandosi come esseri incompresi, puniti solo perché liberi e coraggiosi nel dire la verità:

Io odio questo mio libro. Lo odio con tutto il cuore. Mi ha dato la gloria, quella povera cosa che è la gloria, ma anche tante miserie. Per questo libro ho conosciuto la prigione e il confino, il tradimento degli amici, la malafede degli avversari, l'egoismo e la cattiveria degli uomini. Da questo libro è nata la stupida leggenda che fa di me un essere cinico e crudele, una specie di Machiavelli nei panni del Cardinal di Retz: quando non sono che uno scrittore, un artista, un uomo libero che soffre più dei mali altrui che dei propri ${ }^{68}$.

Anche Céline diviene vittima dei suoi stessi libri, perseguitato a causa dell'insistenza e della violenza con la quale espresse il suo pensiero. L'esilio, la prigione, danno allo scrittore un'ulteriore conferma della meschinità degli uomini e in particolar modo dei francesi, sempre pronti all'attacco, a mettere in ghigliottina, uomini retti da una morale perbenista e intollerante; egli, piuttosto che ammettere le sue colpe e fare un passo indietro riconoscendo la gravità delle leggi antisemite e di quello che stava accadendo sotto i suoi occhi, passò dalla parte di vittima e perseguitato. Tale atteggiamento, come vedremo, riconduce a quello che ebbe Malaparte nei confronti degli italiani dopo il ritorno dall'esilio quando nelle sue ricostruzioni autobiografiche l'autore ribadirà sempre di esser stato accusato e esiliato per antifascismo, per la sua opera letteraria, per aver scritto Tecnica del colpo stato e il Don Camalèo, atteggiandosi, come Céline, a perseguitato politico:

C'è forse qualcuno che mi è grato per quel che ho sofferto per la libertà mia e degli altri? Per il libro che ho scritto in difesa della mia e altrui libertà? Della prigione, della deportazione che ho sofferto per la causa comune della libertà? Per i pericoli che ho incontrato liberamente? No. Coloro per i quali ho sofferto, applaudivano coloro che mi perseguitavano. E una volta liberi, coloro stessi per i quali avevo sofferto mi perseguitarono poi ${ }^{69}$.

Il punto che più accomuna i due scrittori rispetto all'esperienza dell'esilio non è, dunque, semplicemente l'effettiva mancanza di libertà ma soprattutto la diffidenza e il senso di estraneità alla loro nazione che ne deriva. L'epistolario, questo vale anche per Céline, è testimone, infatti, non solo del disconoscimento dell'autore da parte di quel salotto che è il mondo letterario del dopoguerra, ma dell'itinerario di una coscienza individuale che si scontra con l'isolamento, il pregiudizio, la critica di un mondo che sembra non essere mutato dall'epoca precedente. Il loro esilio diviene una condizione interiore. 


\section{Il viaggio in Urss}

69 Céline e Malaparte furono quindi personalità molto versatili e dinamiche, la loro indagine circa la situazione economica e sociale non partiva mai da giudizi a priori, viaggiarono per vedere in faccia i problemi, perché la loro critica ideologica avesse un valore pragmatico.

70 Almeno per quel che concerne la rivoluzione russa, l'ideologia di Céline non deve esser vista come adesione a un partito o a un gruppo, ma come volontà di testimonianza di un mondo colpito dalla cecità e dall'individualismo. Bisogna sempre seguire la sua attitudine di pensatore che si vuole a parte, solitario. In Mea culpa la vicinanza al popolo è da lui analizzata come un'enorme farsa, semplice ipocrisia dell'élite intellettuale che in realtà è del tutto distaccata dal proletariato. Egli non vede nessuna attrattiva nelle case di cultura, vi scopre la stessa falsità dell'intellettualismo francese.

71 Malaparte, al contrario, mostrò sempre un interesse particolare nei confronti del comunismo e anche lui fa quindi del viaggio in Urss un'esperienza fondamentale per comprendere gli sviluppi della storia di quegli anni. Si reca a Mosca nel maggio del 1929 mentre era direttore della Stampa, per approfondire i mutamenti nella psicologia del popolo russo conseguenti alla rivoluzione. Al ritorno pubblicò gli articoli, riuniti in volume nel 1930 con il titolo Intelligenza di Lenin, nel quale, nonostante torni la sua spregiudicatezza, il suo giudizio si fa più concreto e moderato mostrandoci come davvero egli sappia andare al di là delle concezioni fasciste e si apra ad orizzonti mentali e culturali molto diversificati; come Céline, vi esprime un cinismo forte e dure critiche, ma la visione che ne viene fuori è del tutto diversa; rispetto al Mea Culpa lo scritto mostra un'obbiettività che in Céline è difficilmente rintracciabile. Dà prova di essere un osservatore scomodo ma acuto, attento e intelligente. Con grande perspicacia comprende la portata storica dei fenomeni rivoluzionari dell'età moderna, capendo la genesi del profondo mutamento in corso. I toni che egli mantiene sono però sempre lucidi, attraverso uno stile elegante che risulta del tutto opposto all'enfasi degli sfoghi céliniani. Quello che lo accomuna all'analisi di Céline è invece la mancanza di sentimentalismo, il popolo russo è osservato senza falso buonismo e Malaparte crede che dietro tale organizzazione vi sia non comunione basata sul concetto di eguaglianza ma una forte indifferenza per gli altri; col comunismo si è venuto a creare un sistema in cui «ognuno vive estraneo e indifferente alla vita degli altri» $»^{70}$, guidato da freddo egoismo e da indifferenza per i dolori e le gioie altrui; l'esistenza in comune non genera coesione ma individualismo. Anche lui, inoltre, insiste sul fatto che la rivoluzione non era nata in nome della vera libertà ma, al contrario, per far sì che si imponesse il potere del proletariato: potere e libertà non possono convivere. La critica malapartiana è ben aspra e cruda, anche nel suo caso nessuna falsa speranza persiste.

\section{Conclusioni}

Seppur non si possa negare l'evidenza del divario tra l'esperienza dei due scrittori e la singolarità dei loro casi letterari (tanto che il biografo Maurizio Serra, col quale ovviamente non si concorda, definisce "largamente improprio» $»^{71}$ il confronto tra i due) e benché il contatto diretto sia stato quasi inesistente, si ritiene che tale tentativo di comparazione sia invece di particolare interesse, anche a causa della mancanza di studi esistenti sull'argomento ${ }^{72}$. Liberandoci da giudizi preconcetti e riduttivi si è voluto 
quindi tracciare un confronto con vari aspetti della loro opera, provando a delineare un discorso che rendesse la loro complessità e che affrontasse le tematiche comuni.

La loro scrittura, piena di angoscia ma sempre ironica, è un grido contro la falsità: la relazione tra letteratura e critica politica e sociale è fondamentale. In queste pagine ho voluto mostrare come essi rigettarono le classificazioni schematiche scagliandosi sempre contro la borghesia, il falso eroismo, la facile retorica. Furono uomini cinici e freddi, ma estremamente nostalgici e sensibili. La loro unicità letteraria sta nel rifiuto delle convenzioni e dei luoghi comuni: «l'essere un irregolare davanti a qualsiasi regolarità, secondo un desiderio di libertà conservato anche durante il fascismo ${ }^{73}$ è stato scritto di Malaparte.

Partendo dal presupposto teorico di una loro possibile analogia su certi aspetti, attraverso l'analisi di alcuni loro romanzi e saggi, si è provato a dare una consistenza analitica alle ipotesi iniziali. Attraverso una lettura che voleva indagare in primo luogo le analogie del pensiero politico sono stati individuati anche altri aspetti comuni che oltrepassano tali confini e che ci conducono alle loro riflessioni esistenziali: «il pessimismo di Malaparte ci conduce a Céline, alla sua idea di distruzione e all'ossessionante pensiero della morte $»^{74}$. Torna infatti la presenza della morte come vuoto e precarietà, l'angoscia del destino che sovrasta l'uomo, riflessione fondamentale in Voyage au bout de la nuit e invece più celata nei romanzi di Malaparte. La vita è una luce che necessariamente finisce nel buio totale e la notte nei loro romanzi torna ossessivamente come metafora di morte. Morte come paura della fine di tutto, come disperazione intima, come consapevolezza del proprio infimo destino di uomo, come angoscia del tempo che passa:

[...] où on trouvera la force pour ces démarches imbéciles, ces mille projets qui n'aboutissent à rien. Ces tentatives pour sortir de l'accablante nécessité, tentatives qui toujours avortent, et toutes pour aller se convaincre une fois de plus que le destin est insurmontable, qu'il faut retomber au bas de la muraille, chaque soir, sous l'angoisse de ce lendemain, toujours plus précaire, plus sordide ${ }^{75}$.

Anche Malaparte confessa: «il pensiero della morte mi ha dominato fin da bambino, e solo in questi ultimi anni son riuscito a liberarmi da quella dolce e crudele schiavitù ${ }^{76}$, e nell'Albero vivo, inoltre, scrive:

É questa l'ora della nostra morte quotidiana, l'istante in cui il proprio destino appare all'uomo una legge estranea alla sua vita, qualcosa di staccato da lui, senza alcun potere sulla sua coscienza e sulla sua fortuna. Ogni giorno, a quest'ora, noi cominciamo a morire. Questa morte del tempo e della natura, questo universale tramonto, non avviene fuori di noi, ma nel profondo del nostro spirito. La luce si spegne lentamente ${ }^{77}$.

Cercando la comparazione sono quindi saltati fuori gli aspetti generali, storici, culturali, politici, contestuali e tutto ciò ha portato, di conseguenza, ad una migliore conoscenza di ognuno degli scrittori nella sua singolarità. Questa possibilità di connessione che non sminuisce ma arricchisce, che non vuole forzare la similitudine cancellando le singolarità ma al contrario approfondire rifuggendo facili conclusioni, è uno degli aspetti più entusiasmanti e stimolanti della letteratura comparata. 


\section{NOTE}

1. La coerenza logica del percorso malapartiano è difesa, ad esempio, da G. Pardini, in Curzio Malaparte, Biografia politica, Milano, Luni editrice, 1998.

2. L.-F. Céline, Voyage au bout de la nuit [1932], Paris, Gallimard, «Folio», 1972, p. 212.

3. C. Malaparte, Il mio uomo, 1953, Documento autobiografico, in C. Malaparte, La pelle [1949], Milano, Mondadori, 1978, p. 295.

4. Lettera del 19 novembre 1947. Il Camus di cui scrive è un medico amico di entrambi gli scrittori: M. Serra, Malaparte, vite e leggende, Venezia, Marsilio, 2012, p. 415.

5. P. Descaves, «Kaputt» par Malaparte, «Tel Quel», 20 agosto 1946, in M. Serra, op. cit., p. 383.

6. O. Tamburi, Malaparte come me, Milano, Editoriale nuova, 1980, p. 90.

7. S. Freud, Considerazioni attuali sulla guerra e sulla morte, Roma, Newton Compton, 1976, pp. 20-21. Sulle riflessioni di Freud sulla guerra si veda inoltre il carteggio S. Freud, A. Einstein, Perché la guerra?, Torino, Bollati Boringhieri, 1997.

8. G. Grana, Curzio Malaparte, Milano, Marzorati, 1961, p. 63.

9. Si vedano le pagine de La pelle in cui Malaparte descrive la crudele morte di Febo e le pagine di D'un château l'autre in cui Céline descrive la bellezza dell'agonia della sua cagna nella sua casa a Meudon.

10. Su questo aspetto, e non solo, i due autori possono farci pensare a Pierre Drieu La Rochelle, quando, per esempio, scrive: «La décadence, toujours la décadence... La vie est une perpétuelle décadence depuis le début», in Les Chiens de paille, Paris, Gallimard, 1964, p. 200.

11. H. Barbusse, Le Feu [1916], Paris, Albin Michel, 1949, p. 378.

12. L.-F. Céline, Voyage au bout de la nuit, cit., p. 13.

13. C. Malaparte, La rivolta dei santi maledetti [1921], in Id., Opere scelte, a cura di Luigi Martellini, Milano, Mondadori, 1997, p. 25.

14. L.-F. Céline, Voyage au bout de la nuit, cit., p. 94.

15. C. Malaparte, Ritratto delle cose d'Italia, degli eroi, del popolo, degli avvenimenti, delle esperienze e inquietudini della nostra generazione, in Id., L'Europa vivente [1923], Firenze, Vallecchi, 1961, p. 174.

16. Curzio Suckert decise di dar sfogo alle sue idee politiche e ritenne che la lotta dovesse essere svolta aderendo al PNF e iscrivendosi al fascio di Firenze il 20 settembre 1922.

17. C. Malaparte, La rivolta dei santi maledetti, cit., p. 35: «messa in cospetto della notte vergine ed eterna, l'umanità fu presa d'orrore e di spavento, si misurò con quel mistero non umano e ritrovò così la sua essenza più profondamente umana».

18. L.-F. Céline, Voyage au bout de la nuit, cit., p. 33.

19. C. Malaparte, Une declaration obligatoire, in Il Sole è cieco [1947], Paris, Gallimard, «Folio bilingue», 2000, p. 38.

20. C. Malaparte, La rivolta dei santi maledetti, cit., p. 28.

21. L.-F. Céline, Bagatelles pour un massacre, Paris, Denoël, 1937, p. 216.

22. H. Barbusse, op. cit., p. 98.

23. Queste le parole con cui Malaparte spiega il movimento: «[...] L'Oceanismo è un movimento di reazione allo sgretolamento, al particolarismo, all'artificiosità, che oggi rodono le basi della vita, anche cotidiana. La sete del denaro, della speculazione, della lussuria, del provvisorio ha invaso l'umanità [...]», in G.-B. Guerri, L'arcitaliano, vita di Curzio Malaparte, Milano, Bompiani, 1980, p. 37.

24. L.-F. Céline, Voyage au bout de la nuit, cit., pp. 123-124.

25. C. Malaparte, La pelle, cit., p. 60.

26. La scrittura di Louis-Ferdinand Céline non può rientrare, ad esempio, nella definizione di populismo data da Alberto Asor Rosa secondo cui: «L'uso del termine populismo è legittimo solo 
quando sia presente nel discorso letterario una valutazione positiva del popolo, sotto il profilo ideologico oppure storico-sociale oppure etico. Perché ci sia populismo, è necessario insomma che il popolo sia rappresentato come un modello» (A. Asor Rosa, Scrittori e popolo. Saggio sulla letteratura populista in Italia, Roma, Samonà e Savelli, 1965, p. 19).

27. M.-C. Bellosta, Céline ou l'art de la contradiction. Lecture de "Voyage au bout de la nuit», Paris, Cnrs éditions, 2011, p. 238.

28. L.-F. Céline, Voyage au bout de la nuit, cit., p. 151.

29. Ivi, p. 242.

30. C. Malaparte, Diario di uno straniero a Parigi, Firenze, Vallecchi, 1966, p. 41.

31. M. Serra, op. cit., p. 169.

32. C. Malaparte, La pelle, cit., p. 28.

33. L.-F. Céline, Lettre à Évelyne Pollet du 5 juin 1933, in Cahiers Céline 5, Lettres à des amis, a cura di C. Nettelbeck, Paris, Gallimard, 1979, p. 166.

34. C. Malaparte, La pelle, cit., p. 23.

35. Lettera del 1932 a L. Daudet, in F. Vitoux, Louis-Ferdinand Céline, Misère et parole, Paris, Gallimard, 1973, p. 209.

36. M. Serra, op. cit., p. 336. La Germania sconfitta di Céline somiglia molto all'Italia 'liberata' descritta da Malaparte: la trilogia céliniana D'un château l'autre, Nord, Rigodon, come La pelle descrivono un mondo ridotto in macerie con una scrittura colma di orrido. Tale parallelismo è stato rintracciato anche da Luigi Martellini che a tale proposito scrive: «così Napoli è il simbolo della decadenza di tutta l'Europa (quella della trilogia di Céline), il cui marciume trova l'ingorgo finale nell'imbuto di questa città» (Introduzione a C. Malaparte, Opere scelte, cit., p. LXIX).

37. C. Malaparte, La pelle, cit., p. 60.

38. Ivi, p. 27.

39. L.-F. Céline, Voyage au bout de la nuit, cit., p. 74.

40. C. Malaparte, La pelle, cit., p. 38.

41. Ivi, p. 40.

42. M. Kundera, Un incontro, trad. di M. Rizzante, Milano, Adelphi, 2009 (2009), p. 173.

43. L.-F. Céline, Voyage au bout de la nuit, cit., p. 200- 201.

44. C. Malaparte, Diario di uno straniero a Parigi, cit., p. 58.

45. Sulla comparazione tra Céline e Gadda, si veda E. Ferrero, Invito alla lettura di Carlo Emilio Gadda, Milano, Mursia, 1972. Sempre su tale comparazione molto dettagliato è il saggio di Simone Giusti, Gadda verso Céline, in S. Giusti, La congiura stabilita. Dialoghi e comparazioni tra Ottocento e Novecento, Milano, Franco Angeli editore, 2005. Vedi anche il Voyage di Céline e il Pasticciaio di Gadda: due percorsi dall'allegoria alla tautegoria, in L'alternativa letteraria del '900: Gadda, Roma, Savelli, 1975, p. 101-142.

46. G. Contini, Quarant'anni di amicizia, scritti su Carlo Emilio Gadda, (1934-1988), Torino, Einaudi, 1989, p. 25-26.

47. M. Serra, op. cit., p. 75.

48. G. Grana, op. cit., p. 100.

49. M. Serra, op. cit., p. 337.

50. C. Malaparte, Mamma marcia, Firenze, Vallecchi, 1959, p. 88.

51. C. Malaparte, La pelle, cit., p. 110.

52. L.-F. Céline, Voyage au bout de la nuit, cit., p. 49.

53. C. Bo, Introduzione a L.-F. Céline, Morte a credito, traduzione di G. Caproni, Milano, Garzanti, 1981, p. VIII. Per comprendere il suo rapporto col naturalismo cfr. L.-F. Céline, Hommage à Zola, «Études françaises», vol. xxxıx, $n^{\circ}$ 2, 2003: «Aujourd'hui, le naturalisme de Zola, avec les moyens que nous possédons pour nous renseigner, devient presque impossible. On ne sortirait pas de prison si on racontait la vie telle qu'on la sait, à commencer par la sienne. Je veux dire telle qu'on la comprend depuis une vingtaine d'années. Il fallait à Zola déjà quelque héroïsme pour montrer 
aux hommes de son temps quelques gais tableaux de la réalité. La réalité aujourd'hui ne serait permise à personne. À nous donc les symboles et les rêves ! Tous les transferts que la loi n'atteint pas, n'atteint pas encore! Car, enfin, c'est dans les symboles et les rêves que nous passons les neuf dixièmes de notre vie, puisque les neuf dixièmes de l'existence, c'est-à-dire du plaisir vivant, nous sont inconnus, ou interdits. Ils seront bien traqués aussi les rêves, un jour ou l'autre. C'est une dictature qui nous est due».

54. Lettera a Clément Camus, 5 luglio 1950, Bullettins de la bibliotèque de littérature française contemporaine de l'université Paris 7, 1978, Textes et documents III, p. 158, in M.-C. Bellosta, op. cit., p. 311.

55. «Nous travaillons à présent par la sensibilité et non plus par l'analyse, en somme "du dedans"» dice Céline nell'Hommage à Zola, cit., p. 87-91.

56. L. Martellini, Invito alla lettura di Curzio Malaparte, Milano, Mursia, 1977, p. LXXI.

57. L.-F. Céline, Entretiens avec le professeur Y, Paris, Gallimard, 1955, p. 93.

58. D. Aebersold, Céline, un démystificateur mythomane, Paris, Archives des Lettres modernes, 1979, p. 68.

59. G. Grana, op. cit., p. 20.

60. F. Germinario, Céline, letteratura politica e antisemitismo, Torino, Utet, 2011, p. XIX.

61. Sui rapporti tra Malaparte e Maccari vedi Uno strano selvaggio, in G.-B. Guerri, op. cit., pp. 83-96. Sull'argomento molto interessante è inoltre E. Mattiato, Paul Morand e Malaparte. Le tentazioni del cosmopolitismo, in La «Bourse des idées du monde», Malaparte e la Francia, sous la direction de M. Grassi, Firenze, Olschki, 2008, pp. 125-150.

62. F. Germinaro, op. cit., p. XXI.

63. Cfr. É. Séebold, Essai de situation des pamphlets de Louis-Ferdinand Céline, Du Lérot, Tusson, 1985.

64. L.-F. Céline, Bagatelles pour un massacre, Paris, Denoël, 1937, p. 243.

65. J. Morand-Devillier, Les idées politiques de Louis-Ferdinand Céline, Paris, Librairie générale de droit et de jurisprudence, 1972, p. 204.

66. Curzio Malaparte aux îles Lipari, «Le Jour», 16 novembre 1933, in M. Serra, op. cit., p. 211.

67. M. Serra, op. cit., p. 18.

68. C. Malaparte, Che a difendere la libertà ci si rimette sempre, prefazione di Tecnica del colpo di Stato [1948], in Id., Opere scelte, cit., p. 113.

69. Ivi, pp. 75-76.

70. C. Malaparte, Intelligenza di Lenin, Milano, Fratelli Treves editori, 1930, p. 92.

71. M. Serra, Volti e maschere di un esteta armato, Introduzione all'edizione italiana di Id., op. cit., p. 16. Nel 2011 sono sorte delle polemiche riguardanti il caso Céline per le mancate celebrazioni ufficiali del cinquantenario della morte dello scrittore, in tale occasione la stampa francese ha tracciato un confronto col caso Malaparte, di cui era appena uscita in Francia la biografia di Maurizio Serra. È a tal proposito che Serra giudica il confronto inadeguato.

72. A. Arndt, Ungeheure Größen: Malaparte-Céline-Benn. Wertungsprobleme in der deutschen, französischen und italienischen Literaturkritik [Grandezze mostruose: Malaparte - Céline - Benn. Il problema dei 'valori' nella critica letteraria tedesca, francese e italiana], De Gruyter, 2005. Questo, l'unico lavoro dedicato a una comparazione tra Céline e Malaparte in Germania consiste in un confronto tra Malaparte, Céline e l'autore tedesco Benn. Purtroppo, però, non è disponibile nessuna traduzione del saggio. Qualche saggista o giornalista ha accennato, inoltre, a dei punti in comune tra i due scrittori ma mai si è andato a fondo in tale comparazione.

73. L. Martellini, Introduzione, in Malaparte, Opere scelte, cit., p. LV.

74. Ivi, p. LXXII.

75. L.-F. Céline, Voyage au bout de la nuit, cit., p. 200.

76. C. Malaparte, Il giardino perduto, in Id., Opere scelte, cit., p. 322.

77. C. Malaparte, L'albero vivo, in Id., Opere scelte, cit., p. 368. 


\section{RIASSUNTI}

Questo articolo è una ricerca di analogie nelle opere di Curzio Malaparte e Louis-Ferdinand Céline, ricerca che vuole evitare le sterili categorizzazioni in cui i critici spesso hanno incasellato i due autori. Si tratta di un'analisi speculare, pertinente anche a causa della mancanza di studi sull'argomento, che segue un'interpretazione personale della loro opera: tra i due autori c'è un'analogia nella critica diretta alla società capitalista e nell'illustrazione della decadenza del mondo occidentale. Attraverso questa prospettiva si è andati alla ricerca di assonanze e dissonanze tra due scrittori asistematici e inclassificabili.

Cet article est une recherche d'analogies dans l'œuvre de Curzio Malaparte et Louis-Ferdinand Céline qui veut éviter les stériles catégorisations dans lesquelles plusieurs fois les deux auteurs ont été enfermés. C'est une analyse spéculaire, pertinente également à cause du manque d'études sur ce sujet, qui suit une interprétation personnelle de leur œuvre : les deux auteurs ont un point de vue similaire sur la société capitaliste et la décadence du monde occidental. À travers cette perspective on est donc allé à la recherche des assonances et des dissonances entre deux écrivains asystématiques et inclassables.

This essay is a search for analogies in the works of Curzio Malaparte and Louis-Ferdinand Céline. Its main goal is to avoid the sterile and narrow categories into which critics have often confined them. The specular analysis, pertinent also because of the lack of studies on this subject, rests on a personal interpretation of the work of these two authors, who have a similar point of view on capitalistic society and on the decadence of the world. This common postulate explains why it is possible to find so many assonances and dissonances in these two unclassifiable and unsystematic writers.

\section{INDICE}

Mots-clés : guerre, désenchentement, nuit, peuple, dictature, mort, misanthropie, communisme Keywords : war, disenchantment, night, people, dictatorship, death, misanthropy, communism Parole chiave : guerra, disincanto, notte, popolo, dittatura, morte, misantropia, comunismo 